\title{
CARACTERIZACIÓN DE LOS AGENTES EDUCATIVOS EN COLOMBIA
}

\section{CHARACTERIZATION OF EDUCATIONAL AGENTS IN COLOMBIA}

\section{Leidy Carolina Cuervo ${ }^{1}$}

\section{Alix Casadiego Cabrales ${ }^{2}$}

\section{Alvaro Avendaño Rodríguez ${ }^{3}$,}

\section{Luis Ferney Tovar Pérez ${ }^{4}$,}

Universidad Surcolombiana, Neiva $(\mathrm{H})$,

Colombia

$1 \quad$ Licenciada en Pedagogía Infantil- Universidad Surcolombiana, Mg. en educación Universidad Surcolombiana, Doctora en Investigación y Docencia-UNADE-México, Vicerrectora de Investigación y Proyección ORCID https:// orcid.org/0000-0002-6927-0776 carolina.cuervo@usco.edu. co, tel. (057) 8645141

2 Licenciada en Pedagogía Infantil- Universidad Surcolombiana, Mg. en educación Universidad Surcolombiana, Doctora en Sociedad del Conocimiento, Universidad Internacional de la Rioja. ORCID http://orcid.org/0000-00034376-5874 alix.casadiego@usco.edu.co, tel. (057) 8754753 b

3 Mg. Desarrollo Comunitario, Universidad Surcolombiana, Docente de planta, Universidad Surcolombiana ORCID https://orcid.org/0000-0003-0300-400X alave@ usco.edu.co, tel. (057) 8754753

$4 \quad$ Mg. en ecología y gestión de ecosistemas estratégicos, estudios de Doctorado educación y cultura ambiental, Docente catedrático, Universidad Surcolombiana. ORCID https://orcid.org/0000-0001-6805-9760 lufetope@ usco.hotmail.com, tel. (057) 8754753

\section{Resumen}

El presente estudio tiene como finalidad caracterizar los agentes educativos que atienden a la primera infancia en Colombia, de tal forma que se tenga una pauta inicial para diseñar planes de acción en torno a las directrices en materia de educación infantil. Mediante encuestas se recogieron los datos en 8 departamentos de Colombia, encontrando un $23 \%$ de los agentes educativos con edades entre los 31 y 40 años. Así mismo el $66 \%$ no posee estudios universitarios y la mayoría de ellos lo constituyen las madres comunitarias. Finalmente, se concluye sobre los aportes de la investigación para realizar capacitaciones 
adecuadas de acuerdo al perfil de los diferentes agentes educativos, y de esta manera lograr contribuir al desarrollo integral de la primera infancia.

PALABRAS CLAVE: Primera infancia, desarrollo humano, desarrollo infantil, atención integral a la primera infancia, política pública de primera infancia

SUMMARY: The purpose of this study is to characterize the educational agents that attend to early childhood in Colombia, so that there is an initial guideline to design action plans around the guidelines on early childhood education. Surveys collected data in 8 Colombian departments, finding $23 \%$ of educational agents between the ages of 31 and 40 . Likewise, $66 \%$ do not have university studies and most of them are community mothers. Finally, it concludes on the contributions of the research to carry out adequate training according to the profile of the different educational agents, and in this way manage to contribute to the integral development of early childhood.

KEYWORDS: Early childhood, human development, child development, comprehensive early childhood care, early childhood public policy

\section{INTRODUCCIÓN}

Desde los años setenta la prestación de servicios de educación y atención para la primera infancia comenzaron a tener mayor presencia, tanto en el terreno normativo como en el terreno presupuestario en muchas de las políticas educativas estatales de los países iberoamericanos, (Arrabal, 2008).

Actualmente el desarrollo de la primera infancia forma parte del Informe de síntesis del Secretario General de la ONU, el camino hacia la dignidad para 2030, que reconoce la importancia de la primera infancia en la agenda transformativa, acordando "Garantizar una educación inclusiva, equitativa y de calidad y promover oportunidades de aprendizaje durante toda la vida para todos" (Raikes, 2015). Los tiempos actuales imponen retos vertiginosos a los profesionales que trabajan con la infancia, en los escenarios clínico y educativo, no solo en Colombia sino en toda Latinoamérica, (Arango, 2019).

Cada país ha asumido el reto de priorizar la atención a la primera infancia, es una tarea que implica ante toda caracterizar la actual situación de los agentes formadores de tal forma que se pueda garantizar que los niños estén a cargo de personal idóneo y suficiente.

En Suecia, Todas las diferentes profesiones dentro del preescolar son bienvenidas $y$ el personal colabora para combinar sus competencias; las escuelas preescolares se basan en la democracia y, por lo tanto, se considera que todos tienen el mismo valor en el equipo de trabajo, aunque tengan una educación cualitativamente diferente, (Kuisma y Sandberg, 2008).

Canadá permitió recoger argumentos relacionados con la comprensión de que una política nacional de primera infancia, posibilita un proceso de desarrollo humano en el país, (Presidencia de la república, 2013).

En los Estados Unidos, la administración Bush dio a conocer su iniciativa para la primera infancia Good Start, Grow Smart en enero de 2002, la cual estableció nuevas regulaciones para el desarrollo profesional de los primeros educadores, (Wagner y French, 2010).

En Chile, las profesoras de educación parvularia pusieron en evidencia los aspectos positivos reflejados en las Didácticas, los cuales aducen a las últimas reformas educativas que se llevaron a cabo en ese país, (Quintero 2018).

Agregando a lo anterior, el programa cubano "Educa a tu hijo" y el programa colombiano 
"Hogares Comunitarios de Bienestar", dirigidos a la educación de la primera infancia, evidencian el plus del vínculo existente entre educación intercultural y la primera infancia, con el fin de fortalecer el desarrollo integral de niños y niñas (Espinel, Agudelo, Sandoval, y Gutiérrez, 2017). Gestionar las emociones y el bienestar personal se convierte en un elemento básico para favorecer el desarrollo integral del alumno, un elemento clave para trabajar con los futuros docentes en su formación inicial (Bisquerra, 2007; López-Goñi y Goñi, 2012, citado en Ávila, 2019).

En materia de Primera infancia, Colombia ha respondido con la estrategia llamada de "Cero a Siempre", que busca aunar esfuerzos de los sectores público y privado, de las organizaciones de la sociedad civil y de la cooperación internacional a favor de esta población garantizándoles en materia de bienestar algunos componentes en salud, nutrición y educación desde el momento de la gestación hasta los cinco años, (Muñoz, 2013). "De Cero a Siempre" es la Estrategia Nacional de Atención Integral a la Primera Infancia, dirigida a promover y garantizar el pleno desarrollo de las niñas y los niños desde su gestación hasta los seis años de edad de acuerdo con su edad, contexto y condición, (Presidencia de la república, 2014).

La Política de Estado para el Desarrollo Integral de la Primera Infancia de Cero a Siempre, se adopta mediante LEY_1804_2016, que deberá ser implementada en todo el territorio nacional por cada uno de los actores oficiales y privados, tanto del orden nacional como local, que tienen incidencia en el proceso, (Congreso de la República, 2016).

Alineados con la Estrategia De Cero a Siempre, el Ministerio de Educación Nacional, el Instituto Colombiano de Bienestar Familiar, y la Fundación Bancolombia, lanzan las estrategias "Transición es una nota" y "La práctica hace al maestro". Estas estrategias tienen como objetivo mejorar la calidad de la educación inicial mediante la garantía de atención integral en el grado transición, el fortalecimiento pedagógico situado a maestros y maestras, y el acompañamiento a las transiciones de las niñas y los niños en el entorno educativo, (Consejería presidencial para la niñez y adolescencia, 2016). Sin embargo, la investigación sobre la transición preescolar parece estar dominada por estudios realizados en economías desarrolladas como los Estados Unidos, Australia y más ampliamente en Europa, con poca investigación en economías en desarrollo, como América Latina (Urbina, 2014, citado en Urbina, 2019).

De acuerdo con el plan de Atención Integral a la Primera Infancia (PAIPI) la integralidad debe lograrse en esfuerzos intersectoriales que involucren no solo al MEN y al ICBF, sino también a los sectores de salud, cultura y recreación, (Presidencia de la república, 2013), es decir, todas estas personas que interactúan de una $u$ otra manera con los niños y están involucrados en la atención integral de niños menores de cinco años deben ejercer el principio de corresponsabilidad que impone la ley tanto a la familia, como al Estado y a la comunidad, estas personas se les llama "agentes educativos" (Ministerio de Educación Nacional, SF). Por lo tanto, se requiere mejorar las capacitaciones y su apropiación por parte de los agentes educativos encargados de la educación inicial, haciendo claridad sobre la atención integral a la infancia, (de Certain, 2009).

Es oportuno señalar que el conocimiento profesional específico del dominio de los maestros de preescolar desempeña un papel importante en la calidad de la educación y en el aprendizaje de los niños pequeños en diferentes áreas, (Barenthien, Lindner, Ziegler, y Steffensky, 2018) y que cada vez resulta más claro lo importante que es el desarrollo de la primera infancia para el éxito a largo plazo de las familias, las comunidades, los países y, 
en definitiva, para lograr un mundo sostenible y pacífico, (Lombardi, 2015). Para Basadre, (2019), la educación comienza desde antes de la concepción, pues los futuros padres deben prepararse para traer hijos al mundo en las mejores condiciones y poder ofrecérselas después a sus hijos.

Según Bernal, Fernández, Flórez, Gaviria, Ocampo, Samper y Sánchez, (2009), los programas de Hogares comunitarios de bienestar en Colombia, tienen un efecto positivo en lo nutricional, en lo cognitivo y en el desarrollo psicosocial, con mejores interacciones en el juego, en niños que han estado al menos 15 meses expuestos al programa, pero negativo en la salud infantil medido por la incidencia de diarrea y enfermedades respiratorias. Sin embargo, el efecto negativo disminuye a medida que aumenta la exposición al programa.

Los saberes y las prácticas pedagógicas construidas con la primera infancia se caracterizan por ser participativas, significativas, contextuadas y humanizantes, constituyendo un tejido que se nutre además de los recursos comunitarios, del entorno y del medio ambiente, para constituirse en un proyecto de transformación social, (Báez, Garzón, Bejarano y Buitrago, 2015),

Por lo tanto, teniendo en cuenta que los niños están rodeados por diversos tipos de agentes educativos que influyen en su desarrollo integral, y que se hace necesario aunar esfuerzos para trabajar en forma integral para la educación del niño, el presente estudio tiene como objetivo caracterizar los agentes educativos que atienden a la primera infancia en Colombia, de tal forma que se tengan pautas que contribuyan en el diseño de planes de acción en torno a las directrices en materia de educación infantil.

\section{MÉTODO}

El instrumento utilizado en esta investigación fue la encuesta sociodemográfica a 2642 Agentes
Educativos de los diferentes centros de atención a la primera infancia en 8 departamentos de Colombia, que asistieron a capacitación sobre prácticas de cuidado y crianza, con un grupo de 25 tutores. La capacitación fue convocada por el Ministerio de educación nacional, el instituto de bienestar familiar en convenio con la Universidad Surcolombiana.

Se cubrió un total de 69 municipios en los 8 departamentos. La población total de esos municipios es de 5.393.378 de habitantes (11\% de la población colombiana).

Este artículo de investigación científica sistematiza la información recogida previamente, con el fin de obtener una descripción de las características de los Agentes Educativos de esos municipios.

\section{RESULTADOS}

La Figura 1 muestra que el $83 \%$ de los agentes educativos de los diferentes centros de atención a la primera infancia está entre 30 y 60 años, distribuidos aproximadamente en la misma proporción en intervalos de 10 años: $30<40$, $40<50$ y $50<60$.

\section{Distribución por edades}

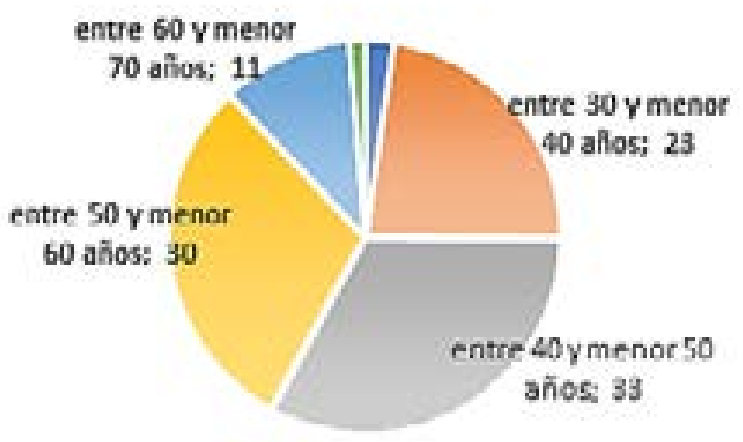

Figura 1 Distribución por edades \%

La Figura 2 muestra que los Agentes Educativos Comunitarios son mayoritariamente mujeres. La proporción de hombres mayores de 30 años como agentes educativos comunitarios en promedio es apenas 3,4\%. 


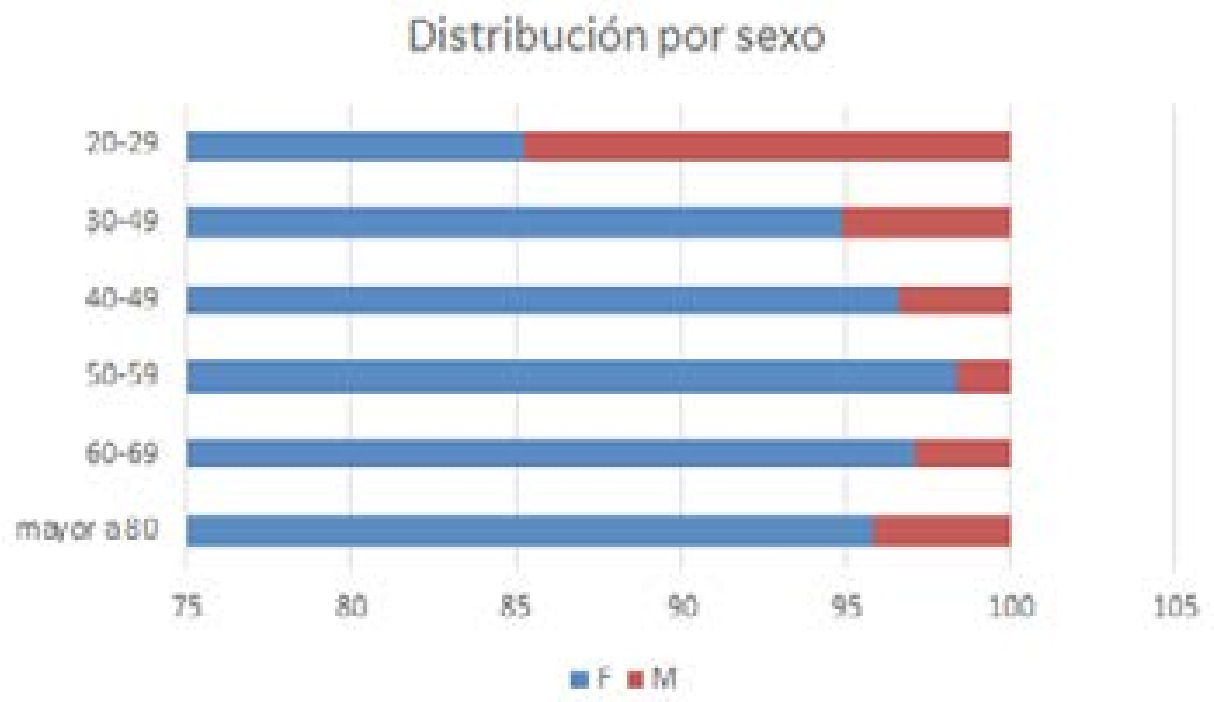

Figura 2 Distribución por sexo

Los 2642 agentes educativos comunitarios que participaron se categorizan en 31 tipos: administrativo, auxiliar administrativo, auxiliar de cuidado del PAIPI, auxiliar pedagógica CDI, auxiliar pedagógico del PAIPI, bacterióloga, bibliotecario, contratista alcaldía oficina de salud, coordinador operativo en la difusión y aplicabilidad de la ley de infancia y adolescencia, coordinador pedagógico PAIPI, delegado primera infancia sector educación departamental, delegado primera infancia sector educación municipal, docente centro desarrollo infantil (cdi), docente de hogar infantil ICBF, docente de transición, docente del PAIPI, enfermero, fonoaudiólogo, formador profesor de música, ludotecario, madre comunitaria ICBF, miembro mesa intersectorial primera infancia, nutricionista, operador primera infancia, promotora de lectura-biblioteca, psicólogo, psicopedagogo, recreacionista, terapeuta ocupacional y trabajador social.

La Figura 3 nos muestra que las madres comunitarias ICBF constituyen aproximadamente la mitad de los agentes educativos. Los 6 tipos de Agentes Educativos Comunitarios mostrados en el gráfico constituyen el $87 \%$ del total. Los otros conforman el restante $13 \%$ cada uno con $3 \%$ o menos.

\section{Tipo de Agente Educativo}

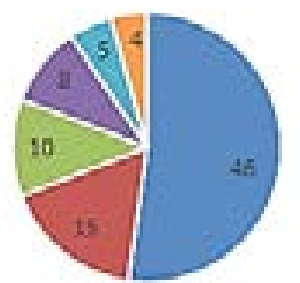

- Madrecomanitario ICBF

- Docente de Hegar Irfontll ICBF

- Doserte de trarsiribn

- Docente Centro Desarrolio infantil(co0

Figura 3 Tipo de Agente Educativo \%

En la Figura 4 tenemos la vista por departamentos. Aquí también las madres comunitarias constituyen la mayoría de los Agentes Educativos Comunitarios. Le siguen los Docentes de Hogar Infantil ICBF y los Docentes del PAIPI. 


\section{Agentes Educativos Comunitarios}

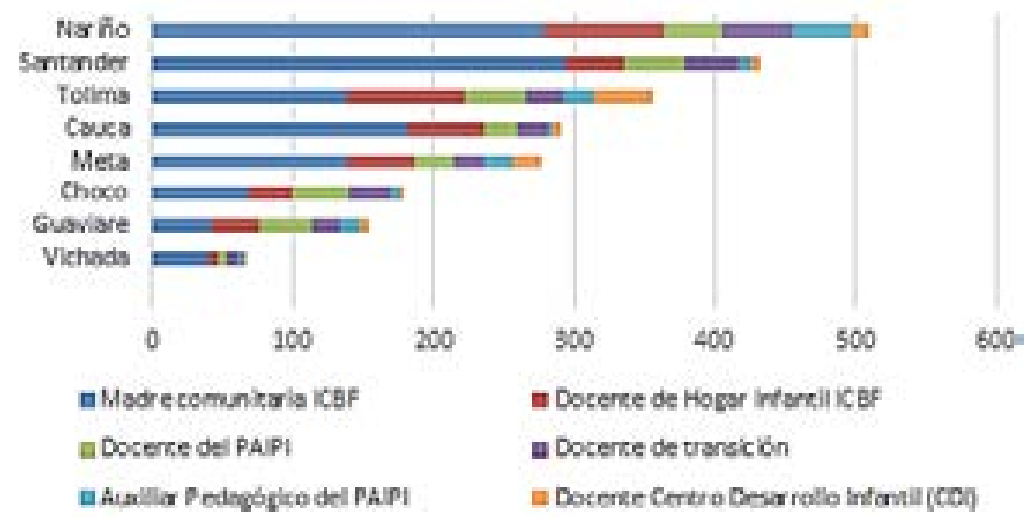

Figura 4 Agentes Educativos Comunitarios por Departamento

La Figura 5 nos indica que un $66 \%$ de los Agentes Educativos Comunitarios no tiene formación superior universitaria ni de postgrado.

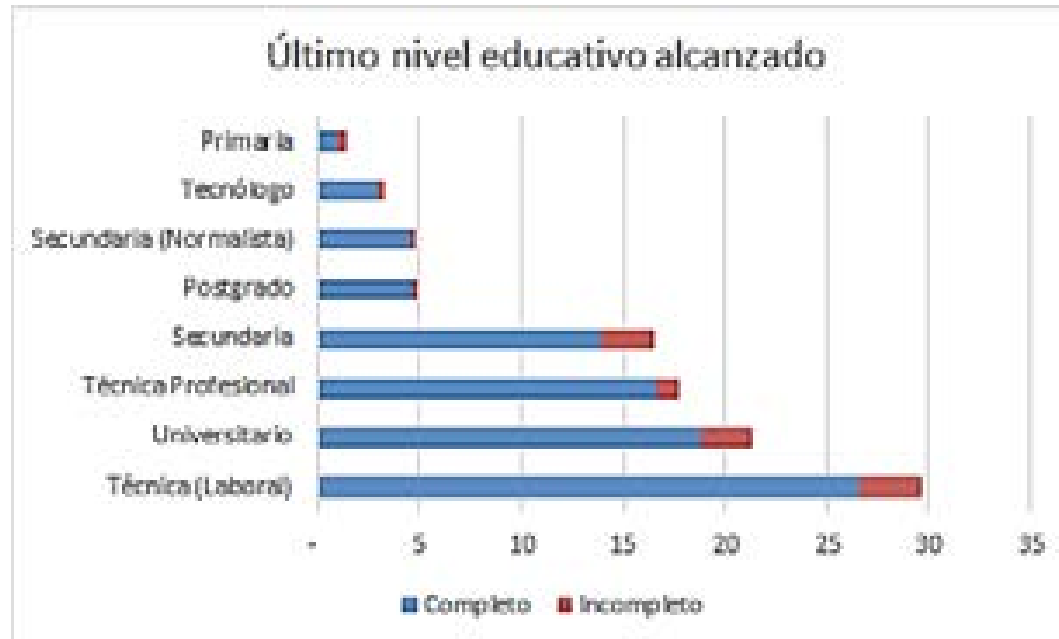

Figura 5 Último nivel educativo alcanzado \%

La Figura 6 nos revela que un $65 \%$ de los Agentes Educativos Comunitarios en edad entre 31 y 40 años tiene formación Técnica (Laboral), Técnica Profesional, Secundaria, Secundaria (Normalista) y Tecnólogo. 


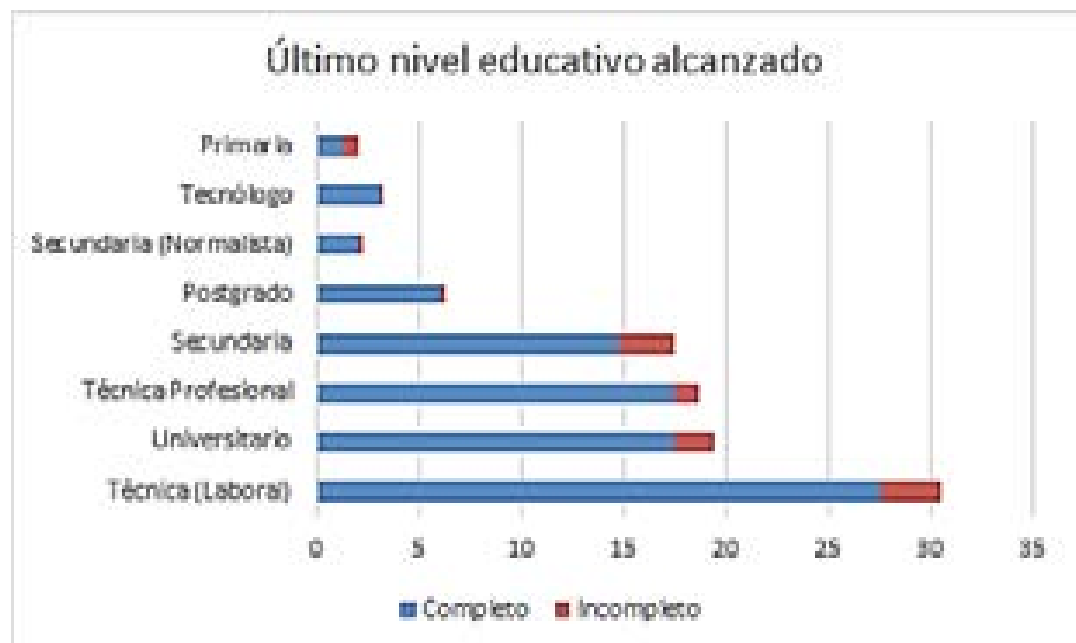

Figura 6 Ultimo nivel educativo entre 31 y 40 años \%

\section{CONCLUSIONES}

El estudio revela que los que los 2642 agentes educativos comunitarios de los 69 municipios encuestados, se categorizan en 31 tipos entre los que encontramos desde administrativos, bibliotecarios, bacteriólogos, fonoaudiólogo, etc. hasta profesores de música, auxiliares pedagógicos, madres comunitarias, docentes de hogares infantiles y docentes de transición, entre otros. Se observa que una buena parte de los agentes educativos lo conforman las madres comunitarias del ICBF. La edad de los Agentes Educativos Comunitarios está entre 30 y 60 años y la mayoría son mujeres. Un $23 \%$ de ellos está entre 31 y 40 años. Así mismo el $66 \%$ no poseen formación superior, lo que revela que las Universidades tienen todavía mucho que aportar en materia de estudios superiores y sería muy provechoso brindarles oportunidades de obtener un título universitario.

Teniendo en cuenta que la atención integral a la primera infancia debe lograrse no solo con la familia y los docentes, sino que también otros sectores como salud, cultura, recreación y en general, todos los que interactúan de diversas formas con los niños, este estudio aporta información valiosa al momento de realizar planes de acción en pro de la primera infancia.
Así mismo, se deben construir capacitaciones más focalizadas de acuerdo al perfil de los diferentes agentes educativos, de tal forma que todos estos sectores tengan los conocimientos básicos para contribuir verdaderamente en la formación de las futuras generaciones.

\section{REFERENCIAS BIBLIOGRÁFICAS}

Raikes, A. (2015) Cómo medir el desarrollo de la primera infancia: prioridades para después de 2015, Espacio para la Infancia, Fundación Bernard van Leer, La Haya, Países bajos

Arango Cardona, S. L. (2019). La experiencia de extrañamiento en las propuestas por la infancia. Revista Boletín Redipe, 8(2), 17-28. Recuperado a partir de https:// revista.redipe.org/index.php/1/article/ view/684

Arrabal,A.A. (2008). Hacia una nueva concepción de la educación de la primera infancia como derecho: avances y desafíos globales. Revista Iberoamericana de educación, 47(5), 1-12.

Ávila Muñoz, A. M. (2019). Perfil docente, bienestar y competencias emocionales para la mejora, calidad e innovación de 
la escuela. Revista Boletín Redipe, 8(5), 131-144. Recuperado a partir de https://revista.redipe. org/index.php/1/article/view/741

Basadre, C. C. (2019). Educación prenatal: una esperanza para el futuro. Revista Boletín Redipe, 8(5), 85-103. Recuperado a partir de https://revista.redipe.org/index.php/1/article/view/739

Barenthien, J., Lindner, M. A., Ziegler, T., \& Steffensky, M. (2018). Exploring preschool teachers' sciencespecific knowledge. Early Years, 1-16.

Bernal, R., Fernández, C., Flórez N., C. E., Gaviria, A., Ocampo, P. R., Samper, B., \& Sánchez, F. (2009). Evaluation of the early childhood program Hogares Comunitarios de Bienestar in Colombia. Available at SSRN 1486209.

Congreso de la República (2016), LEY 1804 DE 2016, Diario Oficial No. 49.953 De 2 De Agosto De 2016

Consejería presidencial para la niñez y adolescencia, (2016) de Cero a Siempre > Prensa > Noticias > $2016>$ Lanzamiento de las estrategias "Transición es una nota" y "La práctica hace al maestro"

de Certain, L. J. (2009). La política de primera infancia y las madres comunitarias. Zona próxima: revista del Instituto de Estudios Superiores en Educación, (11), 86-101.

Diario Oficial No. 46.446 de 8 de noviembre de 2006, Código de la Infancia y la Adolescencia, LEY 1098 DE 2006.

Espinel, C. T. C., Agudelo, L. A. P., Sandoval, E. D. T., \& Gutiérrez, Z. D. P. G. (2017). Proceso de educación intercultural en primera infancia: programa educa a tu hijo-Cuba-y programa hogares comunitarios de bienestar-HBC-Colombia. Praxis Pedagógica, 17(21), 119-142.

Lombardi, J. (2015) Bases para el desarrollo sostenible: el progreso hacia una nueva era para los niños pequeños y las familias, Espacio para la Infancia, Fundación Bernard van Leer, La Haya, Países Bajos

Kuisma, M. y Sandberg, A. (2008). Los pensamientos de los maestros de preescolar y de los estudiantes de preescolar sobre la profesionalidad en Suecia. European Early Childhood Education Research Journal , 16 (2), 186-195.

Ministerio de Educación Nacional, (SF) ¿Quiénes son los agentes educativos? Recuperado de https:// www.mineducacion.gov.co/primerainfancia/1739/article-177854.html

Muñoz,V. J. H. (2013). El ejercicio de ciudadanía desde la primera infancia en Santiago de Cali: una cultura por construir (Master's thesis).

Presidencia de la república, (2013) Estrategia de atención integral a la primera infancia fundamentos políticos, técnicos y de gestión, Alarcón P, Camargo A. y Castro R., (Compiladores) Imprenta Nacional Bogotá D.C., Colombia 2013 
Báez, B. N. Y., Garzón-Rodríguez, J. C., Bejarano-Novoa, D. C., \& Buitrago-Rodríguez, N. E. (2015). Aportes para la educación inicial: saberes construidos por el Proyecto Pedagógico Educativo Comunitario*/Contributions for early education: knowledge constructed by the Pedagogical Educational Community Project/Aportes para a educaçäo inicial na Colômbia: os saberes construídos pelo Projeto Pedagógico Educativo Comunitário. Revista Latinoamericana de Ciencias Sociales, Niñez y Juventud, 13(1), 263.

Quintero, S. (2018). La didáctica en profesores de educación parvularia o preescolar: Los casos de Colombia, V. Revolución en la Formación y la Capacitación para el Siglo XXI, 86.

Presidencia de la república, (2014) Estrategia de atención integral a la primera infancia de cero a siempre informe de balance y prospectiva, Turriago B., Castro R., Peña P., (Compiladores) Edición Carolina Turriago Borrero, Bogotá D.C., Colombia.

Urbina,G. A., (2019). Preschool transition in Mexico: Exploring teachers' perceptions and practices. Teaching and Teacher Education, 85, 226-234.

Wagner, BD y French, L. (2010). Motivación, satisfacción laboral y cambio de maestros entre los maestros de la primera infancia. Revista de Investigación en Educación Infantil, 24 (2), 152-171. 